KCL-MTH-12-10

\title{
Generalised BPS conditions
}

\author{
Peter West \\ Department of Mathematics \\ King's College, London WC2R 2LS, UK
}

\begin{abstract}
We write down two $E_{11}$ invariant conditions which at low levels reproduce the known half BPS conditions for type II theories. These new conditions contain, in addition to the familiar central charges, an infinite number of further charges which are required in an underlying theory of strings and branes. We comment on the application of this work to higher derivative string corrections.
\end{abstract}


The requirement that the solutions which occur in supersymmetric theories preserve a certain amount of the supersymmetry leads to conditions on the charges that they carry. The most obvious brane charges are known to occur as central charges in the supersymmetry algebra [1,2]. As a result, these conditions can be found by insisting that the anti-commutator of the supercharges result in a matrix that has a vanishing determinant, or more precisely, has a certain number of eigenvectors with zero eigenvalues. The most familiar BPS condition, that is derived in this way, relates the space-time momentum to the central charges. Such conditions are known in quite a number of contexts and in particular for black hole solutions they can be found in reference [3].

In this paper we will consider the BPS conditions corresponding to the preservation of half of the supersymmetries (half BPS conditions) in the type II theories. These theories occur at low levels in the non-linear realisation of $E_{11}[4,5,6,7,8,9]$ and the purpose of this paper is to give an $E_{11}$ formulation of these BPS conditions. The brane charges are contained in the first fundamental representation of $E_{11}$ associated with node one, denoted $l_{1}[10,11,12,13,14]$. Hence one would expect the BPS conditions to be a condition on this representation. The $E_{11}$ Dynkin diagram is given by

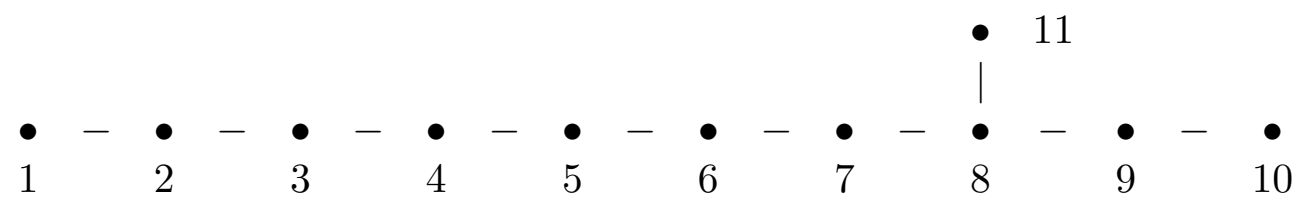

The central charges do not form $U$-duality multiplet and must be complimented by additional charges. By applying U-duality transformations in lower dimensions to known charges, these additional charges have been found sometime ago for certain collections of charges $[15,16,17,18]$; a review of this work can be found in [19]. The $l_{1}$ representation contains the usual central charges but, when viewed from the perspective of lower dimensions, the charges it contains automatically belong to U-duality multiplets. It also provides a higher dimensional origin for all the exotic charges required for this completion. It follows that an $E_{11}$ formulation of the BPS conditions will be a generalisation of the usual BPS conditions, that is the ones derived from the supersymmetry algebra, as they must also contain the additional charges required by U-duality. The half BPS conditions are known to be quadratic in the brane charges and as such we will look for $E_{11}$ invariant conditions that are bilinear in the $l_{1}$ multiplet. We will find two such conditions and show that when they are restricted to contain the familiar charges they will agree with the known half BPS conditions.

As seen from eleven dimensions the $l_{1}$ multiplet contains the objects $[10,11,13]$

$$
\begin{gathered}
P_{\hat{a}}(0), Z^{\hat{a}_{1} \hat{a}_{2}}(1), Z^{\hat{a}_{1} \ldots \hat{a}_{5}}(2), Z^{\hat{a}_{1} \ldots \hat{a}_{7}, b}(3), Z^{\hat{a}_{1} \ldots \hat{a}_{8}}(3), \\
Z^{\hat{b}_{1} \hat{b}_{2} \hat{b}_{3}, \hat{a}_{1} \ldots \hat{a}_{8}}(4), Z^{(\hat{c} \hat{d}), \hat{a}_{1} \ldots \hat{a}_{9}}(4), Z^{\hat{c} \hat{d}, \hat{a}_{1} \ldots \hat{a}_{9}}(4), Z^{\hat{c}, \hat{a}_{1} \ldots \hat{a}_{10}}(4), Z(4) \\
Z^{\hat{c}, \hat{d}_{1} \ldots \hat{d}_{4}, \hat{a}_{1} \ldots \hat{a}_{9}}(5), Z^{\hat{c}_{1} \ldots \hat{c}_{6}, \hat{a}_{1} \ldots \hat{a}_{8}}(5), Z^{\hat{c}_{1} \ldots \hat{c}_{5}, \hat{a}_{1} \ldots \hat{a}_{9}}(5), \\
Z^{\hat{d}_{1}, \hat{c}_{1} \hat{c}_{2} \hat{c}_{3}, \hat{a}_{1} \ldots \hat{a}_{10}}(5), Z^{\hat{c}_{1} \ldots \hat{c}_{4}, \hat{a}_{1} \ldots \hat{a}_{10}}(5,-2), Z^{\hat{a}_{1} \ldots \hat{a}_{11},\left(\hat{c}_{1} \hat{c}_{2}, \hat{c}_{3}\right)}(5), Z^{\hat{a}_{1} \ldots \hat{a}_{11}, \hat{c}, \hat{b}_{1} \hat{b}_{2}}(5), \ldots
\end{gathered}
$$


The indices take the values $\hat{a}_{1}, \hat{b}_{1}, \ldots=1,2, \ldots, 11$. These generators satisfy irreducibility conditions such as $Z^{\left[\hat{a}_{1} \ldots \hat{a}_{7}, \hat{b}\right]}=0, \ldots$. The blocks of eleven indices are inert under SL(11) transformations. As with all representations of $E_{11}$ the states of the $l_{1}$ representation can be classified by a level and the number in brackets gives the level relevant to the eleven dimensional theory. We note that a level $n$ generator has $3 n-1$ indices. The elevendimensional theory emerges from the $E_{11}$ non-linear realisation if one decomposes $E_{11}$ in terms of its SL(11) subalgebra which is the one that emerges if one deletes node eleven in the above $E_{11}$ Dynkin diagram.

In any dimension the $l_{1}$ representation has as its lowest component the space-time momenta $P_{a}, a=1, \ldots, d$, while the form charges in $d$ dimensions are given in the table $[12,13,20]$ below

\begin{tabular}{|c|c|c|c|c|c|c|c|c|c|}
\hline $\mathrm{d}$ & $\mathrm{G}$ & $Z$ & $Z^{a}$ & $Z^{a_{1} a_{2}}$ & $Z^{a_{1} \ldots a_{3}}$ & $Z^{a_{1} \ldots a_{4}}$ & $Z^{a_{1} \ldots a_{5}}$ & $Z^{a_{1} \ldots a_{6}}$ & $Z^{a_{1} \ldots a_{7}}$ \\
\hline 8 & $S L(3) \otimes S L(2)$ & $(\mathbf{3}, \mathbf{2})$ & $\overline{\mathbf{3}}, \mathbf{1})$ & $(\mathbf{1}, \mathbf{2})$ & $(\mathbf{3}, \mathbf{1})$ & $(\overline{\mathbf{3}}, \mathbf{2})$ & $(\mathbf{1}, \mathbf{3})$ & $(\mathbf{3}, \mathbf{2})$ & $(\mathbf{6 , 1})$ \\
& & & & & & & $(\mathbf{8}, \mathbf{1})$ & $(\mathbf{6 , 2})$ & $(\mathbf{1 8 , 1})$ \\
& & & & & & & $(\mathbf{1}, \mathbf{1})$ & & $(\mathbf{3}, \mathbf{1})$ \\
& & & & & & & & & $(\mathbf{6}, \mathbf{1})$ \\
& & $\mathbf{1 0}$ & $\overline{\mathbf{5}}$ & $\mathbf{5}$ & $\mathbf{1 0}$ & $\mathbf{2 4}$ & $\mathbf{4 0}$ & $\mathbf{7 0}$ & - \\
\hline 7 & $S L(5)$ & & & & & $\mathbf{1}$ & $\mathbf{1 5}$ & $\mathbf{5 0}$ & - \\
& & & & & & & $\mathbf{1 0}$ & $\mathbf{4 5}$ & - \\
& & & & & & & & $\mathbf{5}$ & - \\
\hline 6 & $S O(5,5)$ & $\mathbf{1 6}$ & $\mathbf{1 0}$ & $\mathbf{1 6}$ & $\mathbf{4 5}$ & $\mathbf{1 4} 4$ & $\mathbf{3 2 0}$ & - & - \\
& & & & & $\mathbf{1}$ & $\mathbf{1 6}$ & $\mathbf{1 2 6}$ & - & - \\
& & & & & & & $\mathbf{1 2 0}$ & - & - \\
\hline 5 & $E_{6}$ & $\mathbf{2 7}$ & $\mathbf{2 7}$ & $\mathbf{7 8}$ & $\mathbf{3 5} 1$ & $\mathbf{1 7 2 8}$ & - & - & - \\
& & & & $\mathbf{1}$ & $\mathbf{2 7}$ & $\mathbf{3 5 1}$ & - & - & - \\
& & & & & & $\mathbf{2 7}$ & - & - & - \\
\hline 4 & $E_{7}$ & $\mathbf{5 6}$ & $\mathbf{1 3 3}$ & $\mathbf{9 1 2}$ & $\mathbf{8 6 4 5}$ & - & - & - & - \\
& & & $\mathbf{1}$ & $\mathbf{5 6}$ & $\mathbf{1 5 3 9}$ & - & - & - & - \\
& & & & & $\mathbf{1 3 3}$ & - & - & - & - \\
& & & & & $\mathbf{1}$ & - & - & - & - \\
\hline 3 & $E_{8}$ & $\mathbf{2 4 8}$ & $\mathbf{3 8 7 5}$ & $\mathbf{1 4 7 2 5 0}$ & - & - & - & - & - \\
& & $\mathbf{1}$ & $\mathbf{2 4 8}$ & $\mathbf{3 0 3 8 0}$ & - & - & - & - & - \\
& & & $\mathbf{1}$ & $\mathbf{3 8 7 5}$ & - & - & - & - & - \\
& & & & $\mathbf{2 4 8}$ & - & - & - & - & - \\
& & & & $\mathbf{1}$ & - & - & - & - & - \\
\hline
\end{tabular}

The definition of the level depends on the dimension one is in; the level of the charges in $d$ dimensions is the number of upper $d$-dimensional indices they carry.

We begin by finding an $E_{11}$ formulation of the most common BPS condition which is of the generic form $p^{2}+Z^{2}=0$ where $p_{\mu}$ is the space-time momentum and $Z$ one of the 
central charges. Since this is bilinear in the charges and consists of a single equation we are looking for an $E_{11}$ invariant that is bilinear in the $l_{1}$ representation. Let us denote the charges in the $l_{1}$ representation by $l$. Their transformation under an $E_{11}$ group element $g_{0}$ can be written in the form

$$
l^{\prime}=D\left(g_{0}^{-1}\right) l, \quad \text { or in components } \quad l_{N}^{\prime}=D\left(g_{0}^{-1}\right)_{N}{ }^{M} l_{M}
$$

where $D$ are the matrices of the $l_{1}$ representation and $l$ is taken to be a column vector.

The non-linear realisation of $E_{11}$ is constructed from an $E_{11}$ group element $g(\xi)$ where $\xi$ parameterise the group element. In the non-linear realisation the $\xi$ become the fields of the theory. This group element is subject to the transformations

$$
g(\xi) \rightarrow g\left(\xi^{\prime}\right)=g_{0} g(\xi), \quad g_{0} \in E_{11}, \quad \text { as well as } \quad g(\xi) \rightarrow g(\xi) h, \quad h \in I\left(E_{11}\right)
$$

The group element $g_{0} \in E_{11}$ is a rigid transformation, that is, a constant, while $h \in$ $I\left(E_{11}\right)$ is a local transformation. Here $I$ is the Cartan involution and $I\left(E_{11}\right)$ is the Cartan involution invariant subgroup of $E_{11}$ and as a result $I(h)=h$. For a discussion of the Cartan involution and $I\left(E_{11}\right)$ see section 16.7 .3 of [21], or earlier papers on $E_{11}$. We note that the action of the Cartan involution preserves the order of two group elements $g_{1}$ and $g_{2}$, that is, $I\left(g_{1} g_{2}\right)=g_{1} g_{2}$.

Using the element $g(\xi)$ that occurs in the non-linear realisation we can consider the states

$$
L(\xi) \equiv D\left(g(\xi)^{-1}\right) l
$$

It is straightforward to verify that they transform under rigid and local transformation as follows

$$
L^{\prime}=D\left(g(\xi)^{-1}\right) D\left(g_{0}^{-1}\right) l=D\left(g\left(\xi^{\prime}\right)^{-1}\right) l=L\left(\xi^{\prime}\right), \quad \text { and } \quad L^{\prime}=D\left(h^{-1}\right) L(\xi)
$$

In the maximal supergravity theories the supercharges do not transform under the $U$ duality group but under its Cartan involution invariant subgroup and as a result the central charges also transform under this latter group. As such we can expect the BPS conditions to be constructed from $L$.

From the $l_{1}$ representation one can construct what is called the dual twisted representation $l_{I D}$, which transforms as

$$
l_{I D}^{\prime}=l_{I D} D\left(I\left(g_{0}\right)\right), \quad \text { or in components } \quad l_{I D}{ }^{M}=l_{I D}{ }^{N} D\left(g_{0}^{-1}\right)_{N}{ }^{M}
$$

where $l_{I D}$ is taken to be a row vector. Clearly given the matrices $D$ one can define such a transformation and it is trivial to verify that it is indeed a representation. For a more detailed discussion of such representations and some of the other steps above see the appendix B of reference [22]. In fact the dual twisted representation $l_{I D}$ has the same highest weight as the original representation $l$ as the Cartan subalgebra elements are preserved by the combined action of an inverse transformation and the Cartan involution. As such the representation $l_{I D}$ can be identified with the transpose of the original representation, that is, with $l^{T}$. The analog of $L$ for the twisted dual representation is $L_{I D}(\xi)=l^{T} D((I g(\xi)))$ 
Using equation (3) it is trivial to verify that

$$
L^{2} \equiv l_{I D} D(I(g(\xi))) D\left(g(\xi)^{-1}\right) l=l^{T} D(I(g(\xi))) D\left(g(\xi)^{-1}\right) l=l^{T} D\left(M(\xi)^{-1}\right) l=L^{T}(\xi) L(\xi)
$$

is invariant under both the rigid and local transformations of equation, that is, the transformations of the non-linear realisation (3). In the second step we have defined the object $M(\xi) \equiv g(\xi) I\left(g(\xi)^{-1}\right.$.

It will be useful to rewrite the above expression for $L^{2}$. When constructing the nonlinear realisation of the semi-direct product of $E_{11}$ and the generators of the $l_{1}$ representation, denoted by $E_{11} \otimes_{s} l_{1}$, one automatically finds a generalised space-time, whose elements $x^{N}$ are in one to one correspondence with the $l_{1}$ representation, that is equipped with a generalised vielbein $[10,7]$. We will not give an account of this construction here but we will recall the parts we need. For each element of the $l_{1}$ representation we introduce corresponding generators, that we denote by $\tilde{l}$, and which by definition have commutators with the generators of the $E_{11}$ algebra such that for any $g_{0} \in E_{11}$ transformation

$$
g_{0}^{-1} \tilde{l} g_{0}=D\left(g_{0}\right) \tilde{l}, \quad \text { or in components } \quad g_{0}^{-1} \tilde{l}_{N} g_{0}=D\left(g_{0}\right)_{N}{ }^{M} \tilde{l}_{M}
$$

The infinitesimal version of this relation are just the commutators of the $E_{11}$ generators and the $\tilde{l}$ generators. These are given in the appendix. It turns out that the generalised vielbein $E_{N} A$ is given by

$$
g^{-1}(\xi) d x \cdot \tilde{l} g(\xi)=D(g(\xi)) \tilde{l}=d x \cdot E \cdot \tilde{l}
$$

or in components

$$
g^{-1}(\xi) d x \cdot \tilde{l} g(\xi)=d x^{N} D(g)_{N}{ }^{A} \tilde{l}_{A}=d x^{N} E_{N}{ }^{A} \tilde{l}_{A}
$$

The $x^{N}$ are the coordinates of the generalised space-time that arises in the non-linear realisation of $E_{11} \otimes_{s} l_{1}$, but in this paper they will just act as dummy variables. Equation (9) contains the expression that arises in the non-linear realisation and defines the generalised vielbein $E_{N}{ }^{A}$. We recogonise, using matrix notation, that

$$
E=D(g(\xi)), \quad \text { and so } \quad E^{-1}=D\left(g(\xi)^{-1}\right)
$$

Using the known $E_{11}$ commutators with the generators in the $l_{1}$ representations, given in the appendix, it is straightforward to compute the generalised vielbein in any dimension, at least at low levels and below we will give an example.

Substituting equation (9) into equation (7) we find that

$$
L^{2} \equiv l^{T}\left(E^{-1}\right)^{T} E^{-1} l=L_{A} L_{A}
$$

where as previously defined $L_{A}=\left(E^{-1}\right){ }_{A}{ }^{N}$. Technically the transpose acting on a group element is defined by $g^{T}=I\left(g^{-1}\right)$, but the corresponding action on the matrix representative is indeed the transpose. 
We take half BPS states to obey the $E_{11}$ invariant condition

$$
L^{2}=0
$$

It has been found that the half BPS states also obey another condition, or rather a Uduality multiplet of conditions, which are also bilinear in the charges $[15,16,17,18,19]$. As such the $E_{11}$ formulation of this condition should also be bilinear in the $l_{1}$ representation. Guided by the fact that the first component in the U-duality multiplet is an object with one upper space-time vector index we take this condition to be that the tensor product of two $l_{1}$ representations restricted to the fundamental representation associated with node ten, that is $l_{10}$, vanishes. In symbols this is the condition is given by

$$
\left.l_{1} \otimes l_{1}\right|_{l_{10}}=0
$$

Clearly this condition is $E_{11}$ invariant. We note that it only involves the brane charges and not the generalised vielbein and so it does not involve the fields of the theory. When viewed from eleven dimensions the $l_{10}$ representation contains the following objects

$$
\begin{gathered}
S^{\hat{a}}(1,1) ; S^{\hat{a}_{1} \ldots \hat{a}_{4}}(2,1), S^{\hat{a}_{1} \ldots \hat{a}_{7}}(3,1) ; S^{\hat{a}_{1} \ldots \hat{a}_{6}, \hat{b}}(3,1) \\
S^{\hat{a}_{1} \ldots \hat{a}_{10}}(4,1), S^{\hat{a}_{1} \ldots \hat{a}_{9}, \hat{b}}(4,2), S^{\hat{a}_{1} \ldots \hat{a}_{8}, \hat{b}_{1} \hat{b}_{2}}(4,1), S^{\hat{a}_{1} \ldots \hat{a}_{8},\left(\hat{b}_{1} \hat{b}_{2}\right)}(4,1), S^{\hat{a}_{1} \ldots \hat{a}_{7}, \hat{b}_{1} \hat{b}_{2} \hat{b}_{3}}(4,1), \\
S^{\hat{a}_{1} \ldots \hat{a}_{11}, \hat{b}_{1} \hat{b}_{2}}(5,3), S^{\hat{a}_{1} \ldots \hat{a}_{11},\left(\hat{b}_{1} \hat{b}_{2}\right)}(5,3), \\
S^{\hat{a}_{1} \ldots \hat{a}_{10}, \hat{b}_{1} \hat{b}_{2} \hat{b}_{3}}(5,3), S^{\hat{a}_{1} \ldots \hat{a}_{10}, \hat{b}_{1} \hat{b}_{2}, \hat{c}_{1}}(5,3), S^{\hat{a}_{1} \ldots \hat{a}_{10},\left(\hat{b}_{1} \hat{b}_{2} \hat{b}_{3}\right)}(5,1), \\
S^{\hat{a}_{1} \ldots \hat{a}_{9}, \hat{b}_{1} \hat{b}_{2} \hat{b}_{3} \hat{b}_{4}}(5,2), S^{\hat{a}_{1} \ldots \hat{a}_{9}, \hat{b}_{1} \hat{b}_{2} \hat{b}_{3}, \hat{c}_{1}}(5,2), \\
S^{\hat{a}_{1} \ldots \hat{a}_{8}, \hat{b}_{1} \ldots \hat{b}_{5}}(5,1), S^{\hat{a}_{1} \ldots \hat{a}_{8}, \hat{b}_{1} \ldots \hat{b}_{4}, \hat{c}}(5,1), S^{\hat{a}_{1} \ldots a_{7}, \hat{b}_{1} \ldots \hat{b}_{6}}(5,1), \ldots
\end{gathered}
$$

The indices grouped together are anti-symmetrised except where indicated otherwise and the obey the usual irreduciblility constraints, for example $S^{\left[\hat{a}_{1} \ldots \hat{a}_{6}, \hat{b}\right]}=0$. One can find these results analytically using the techniques of references [23,24] which are reviewed in section 16.6.2 reference [21], or by using the Nutma computer programme [25]. The numbers in brackets are the level, appropriate to the eleven dimensional decomposition, and the multiplicity respectively. We have taken the highest weight, that is the first, state to have level one.

To evaluate the components of equation (13) we must construct the $l_{10}$ representation from two $l_{1}$ representations. The first step is to write down objects with the correct SL(11) index structure and level from two $l_{1}$ representations with arbitrary coefficients. The coefficients can be determined by starting with the first component, which is given by $S^{\hat{a}}=P_{\hat{b}} Z^{\hat{b} \hat{a}}$, and varying it under the $E_{11}$ transformation $\Lambda_{\hat{c}_{1} \hat{c}_{2} \hat{c}_{3}} R^{\hat{c}_{1} \hat{c}_{2} \hat{c}_{3}}+\Lambda^{\hat{c}_{1} \hat{c}_{2} \hat{c}_{3}} R_{\hat{c}_{1} \hat{c}_{2} \hat{c}_{3}}$. One finds, using the commutators in the appendix, that

$$
\delta S^{\hat{a}}=\left[\Lambda_{\hat{c}_{1} \hat{c}_{2} \hat{c}_{3}} R^{\hat{c}_{1} \hat{c}_{2} \hat{c}_{3}}+\Lambda^{\hat{c}_{1} \hat{c}_{2} \hat{c}_{3}} R_{\hat{c}_{1} \hat{c}_{2} \hat{c}_{3}}, P_{\hat{b}} Z^{\hat{b} \hat{a}}\right]
$$




$$
=-\Lambda_{\hat{c}_{1} \hat{c}_{2} \hat{c}_{3}}\left(Z^{\hat{c}_{1} \hat{c}_{2} \hat{c}_{3} \hat{a} \hat{b}} P_{\hat{b}}+3 Z^{\hat{a} \hat{c}_{1}} Z^{\hat{c}_{2} \hat{c}_{3}}\right)=\Lambda_{\hat{c}_{1} \hat{c}_{2} \hat{c}_{3}} S^{\hat{a} \hat{c}_{1} \hat{c}_{2} \hat{c}_{3}}
$$

and hence the expression for $S^{a_{1} \ldots a_{4}}$ given below. We note that $Z^{\hat{a}_{1}\left[\hat{a}_{2}\right.} Z^{\left.\hat{a}_{3} \hat{a}_{4}\right]}=Z^{\left[\hat{a}_{1} \hat{a}_{2}\right.} Z^{\left.\hat{a}_{3} \hat{a}_{4}\right]}$. At the next level we have

$$
\begin{aligned}
& \delta S^{\hat{a}_{1} \hat{a}_{2} \hat{a}_{3} a_{4}}=\left[\Lambda_{\hat{c}_{1} \hat{c}_{2} \hat{c}_{3}} R^{\hat{c}_{1} \hat{c}_{2} \hat{c}_{3}}, S^{\hat{a}_{1} \ldots \hat{a}_{4}}\right]=\Lambda_{\hat{c}_{1} \hat{c}_{2} \hat{c}_{3}}\left(-6 Z^{\left[\hat{a}_{1} \hat{a}_{2} \mid \hat{c}_{1} \hat{c}_{2} \hat{c}_{3}\right.} Z^{\left.\mid \hat{a}_{3} \hat{a}_{4}\right]}+3 Z^{\hat{c}_{1} \hat{c}_{2}} Z^{\hat{c}_{3} \hat{a}_{1} \hat{a}_{2} \hat{a}_{3} \hat{a}_{4}}\right. \\
& \left.+P_{\hat{b}} Z^{b \hat{a}_{1} \hat{a}_{2} \hat{a}_{3} \hat{a}_{4} \hat{c}_{1} \hat{c}_{2}, \hat{c}_{3}}+P_{\hat{b}} Z^{b \hat{a}_{1} \hat{a}_{2} \hat{a}_{3} \hat{a}_{4} \hat{c}_{1} \hat{c}_{2} \hat{c}_{3}}\right)=\Lambda_{\hat{c}_{1} \hat{c}_{2} \hat{c}_{3}} S^{\hat{a}_{1} \hat{a}_{2} \hat{a}_{3} \hat{a}_{4} \hat{c}_{1} \hat{c}_{2}, \hat{c}_{3}}+\Lambda_{\hat{c}_{1} \hat{c}_{2} \hat{c}_{3}} S^{\hat{a}_{1} \hat{a}_{2} \hat{a}_{3} \hat{a}_{4} \hat{c}_{1} \hat{c}_{2} \hat{c}_{3}}
\end{aligned}
$$

Taking anti-symmetry in all the indices it is straightforward to read off the expression for $S^{a_{1} \ldots a_{6} a_{7}}$ given below. The expression for $S^{a_{1} \ldots a_{6}, b}$ can also be found after some non-trivial manipulations. We have found that

$$
\begin{gathered}
S^{\hat{a}}=P_{\hat{b}} Z^{\hat{b} \hat{a}} ; S^{\hat{a}_{1} \ldots \hat{a}_{4}}=-3 Z^{\left[\hat{a}_{1} \hat{a}_{2}\right.} Z^{\left.\hat{a}_{3} \hat{a}_{4}\right]}+P_{\hat{b}} Z^{\hat{b} \hat{a}_{1} \hat{a}_{2} \hat{a}_{3} \hat{a}_{4}}, \\
S^{\hat{a}_{1} \ldots \hat{a}_{7}}=-3 Z^{\left[\hat{a}_{1} \hat{a}_{2}\right.} Z^{\left.\hat{a}_{3} \ldots \hat{a}_{7}\right]}+P_{\hat{b}} Z^{\hat{b} \hat{a}_{1} \ldots \hat{a}_{7}}, \\
S^{\hat{a}_{1} \ldots \hat{a}_{6}, \hat{b}}=P_{\hat{c}} Z^{\hat{c} \hat{a}_{1} \ldots \hat{a}_{6}, \hat{b}}+\frac{3.6 .5}{7}\left(Z^{\hat{b}\left[\hat{a}_{1}\right.} Z^{\left.\hat{a}_{2} \ldots \hat{a}_{6}\right]}-Z^{\left[\hat{a}_{1} \hat{a}_{2}\right.} Z^{\left.\hat{a}_{3} \ldots \hat{a}_{6}\right] \hat{b}}\right), \ldots
\end{gathered}
$$

The half BPS condition of equation (13) takes the form

$$
S^{a}=0=S^{\hat{a}_{1} \ldots \hat{a}_{4}}=S^{\hat{a}_{1} \ldots \hat{a}_{7}}=S^{\hat{a}_{1} \ldots \hat{a}_{6}, \hat{b}}=\ldots
$$

We have used the symbol $S$ to denote both the components of the abstract $l_{10}$ representation and the $l_{10}$ constructed from two $l_{1}$ representations. Although we have given the $l_{1}$ and $l_{10}$ multiplets in eleven dimensions one can carry out the usual dimensional reduction process to find the corresponding results in lower dimensions.

We will now evaluate the above half BPS conditions for the type II theory in $d$ dimensions at level zero. The $d$ dimensional theory emerges from the non-linear realisation if we decomposing $E_{11}$ into the algebra $G L(d) \otimes E_{11-d}$. The Dynkin diagram of this algebra remains when we delete node $\mathrm{d}$ in the $E_{11}$ Dynkin diagram and the objects that occur in this decomposition can be classified by a level which is associated with node $d$. The first factor, that is $G L(d)$, corresponds to $d$ dimensional gravity and the second fact is the U-duality symmetry in $d$ dimensions. At level zero we have the graviton in $d$ dimensions and the scalar fields that belong to the non-linear realisation of $E_{11-d}$ while in the $l_{1}$ representation we have the space-time momentum $p_{\mu}$ in $d$ dimensions and the charges, denoted $Z$, that are scalars with respect to the GL(d) symmetry, but transform with respect to the $E_{11-d}$ in the representations listed in the first column of table one. The generalised vielbein at level zero is of the form

$$
E=(\operatorname{det} e)^{-\frac{1}{2}}\left(\begin{array}{cc}
e_{\mu}^{a} & 0 \\
0 & \mathcal{E}^{(0)}
\end{array}\right)
$$

where $e_{\mu}{ }^{a}$ is the vielbein in the $d$ dimensional space-time and $\mathcal{E}^{(0)}$ is the vielbein in the internal sector at level zero, with the factor of $(\operatorname{det} e)^{-\frac{1}{2}}$ taken out. The factor $(\operatorname{det} e)^{-\frac{1}{2}}$ 
drops out of the BPS condition; it has its origins in the unconventional commutator between the generators of GL(d) and the space-time translations which follows from $E_{11}$. As a result the half BPS condition of equation (12) becomes

$$
p^{2}+Z^{T}\left(\mathcal{E}^{(0) T}\right)^{-1}\left(\mathcal{E}^{(0)}\right)^{-1} Z=0
$$

The condition of equation (1.13) also simplifies if we are at level zero. For dimensions $4 \leq d \leq 10$ we can set all objects with any block of anti-symmetrised indices that contains more than seven indices to zero and equation (17) now takes the form

$$
\begin{gathered}
P_{i} Z^{i j}=0, \quad-3 Z^{\left[i_{1} i_{2}\right.} Z^{\left.i_{3} i_{4}\right]}+P_{j} Z^{j i_{1} i_{2} i_{3} i_{4}}=0, \quad-3 Z^{\left[i_{1} i_{2}\right.} Z^{\left.i_{3} \ldots i_{7}\right]}+P_{k} Z^{i_{1} \ldots i_{7}, k}=0 \\
P_{k} Z^{k i_{1} \ldots i_{6}, j}+\frac{3.6 .5}{7}\left(Z^{j\left[i_{1}\right.} Z^{\left.i_{2} \ldots i_{6}\right]}-Z^{\left[i_{1} i_{2}\right.} Z^{\left.i_{3} \ldots i_{6}\right] j}\right)=0, \ldots
\end{gathered}
$$

These agree with the results given in [19].

It is instructive to evaluate the half BPS conditions of equations (20) and (21) explicitly for seven dimensions where $E_{4}=S L(5)$. Apart from the graviton in seven dimensions we have fourteen scalars which belong to the non-linear realisation of SL(5) with local subgroup $\mathrm{SO}(5)$. The latter arise from the eleven dimensional fields as $h_{i}{ }^{j}$ and $A_{i_{1} i_{2} i_{3}}$ where $i, j, \ldots=1,2,3,4$. As such the part of the $E_{11}$ group element $g(\xi)$ in the internal sector and at level zero is given by

$$
g^{(0)}(\xi)=e^{h_{i}{ }^{j} K^{i}} e^{\frac{1}{3 !} A_{i_{1} i_{2} i_{3}} R^{i_{1} i_{2} i_{3}}}
$$

The superscript 0 just indicates that we are at level zero. The charges $Z$ of the $l_{1}$ representation at level zero transform as the 10 of SL(5) and arise from eleven dimensions as $Z=\left\{P_{i}, Z^{i_{1} i_{2}}\right\}$. As a result in the internal sector and at level zero

$$
(d x \cdot l)^{(0)}=d x^{i} P_{i}+d x_{i_{1} i_{2}} Z^{i_{1} i_{2}}
$$

It is almost trivial, using equation (22) and the commutators of the appendix, to verify that the generalised vielbein in the internal sector is given by

$$
\mathcal{E}^{(0)}=\left(\begin{array}{cc}
e_{\bar{i}}^{j} & -\frac{1}{2} e_{\bar{i}}^{k} C_{k i_{i} i_{2}} \\
0 & \left(e^{-1}\right)_{i_{1} i_{2}} \bar{j}_{1} \bar{j}_{2}
\end{array}\right), \quad \text { and } \quad\left(\mathcal{E}^{(0)}\right)^{-1}=\left(\begin{array}{cc}
\left(e^{-1}\right)_{i}{ }^{\bar{j}} & +\frac{1}{2} C_{i k_{i} k_{2}} e_{\bar{j}_{1} \bar{j}_{2}}{ }^{k_{1} k_{2}} \\
0 & e_{\bar{j}_{1} \bar{j}_{2}} i_{1} i_{2}{ }^{2}
\end{array}\right)
$$

where $e_{\bar{i}}{ }^{i}=\left(e^{h}\right)_{\bar{i}}{ }^{i}, e_{\bar{j}_{1} \bar{j}_{2}}{ }^{i_{1} i_{2}}=e_{\bar{j}_{1}}{ }^{\left[i_{1}\right.} e_{\bar{j}_{2}}{ }^{\left.i_{2}\right]}$ and $e^{-1}{ }_{i_{1} i_{2}} \bar{j}_{1} \bar{j}_{2}=\left(e^{-1}\right)_{i_{1}}^{\left[\bar{j}_{1}\right.}\left(e^{-1}\right)_{i_{2}}{ }^{\left.\bar{j}_{2}\right]}$. We are using $\bar{i}, \bar{j}, \ldots$ as world indices in the four dimensional internal space. The construction of the non-linear realisation in seven dimensions at level zero in the internal sector was given in detail in reference [26]. This reference also contains the generalised vielbein in dimensions $4 \leq d \leq 7$. The charges referred to the tangent space, denoted previously by $L$, are then given by

$$
L=\left(\mathcal{E}^{(0)}\right)^{-1} Z=\left(\begin{array}{c}
\left(e^{-1}\right)_{i}{ }^{\bar{j}} P_{\bar{j}}+\frac{1}{2} C_{i k_{i} k_{2}} e_{\bar{j}_{1} \bar{j}_{2}}^{k_{1} k_{2}} Z^{\bar{j}_{1} \bar{j}_{2}} \\
e_{\bar{j}_{1} \bar{j}_{2}}{ }^{i_{1} i_{2}} Z^{\bar{j}_{1} \bar{j}_{2}}
\end{array}\right)
$$

which can be substituted into the half BPS condition of equation (20). 
For the seven dimensional theory the half BPS condition of equation (21) at level zero takes the form

$$
P_{i} Z^{i j}=0
$$

We now consider the half BPS condition when we keep the charges at the next level in the $l_{1}$ representation where we find a charge with a $d$-dimensional vector index which belongs to the representations of $E_{11-d}$ given in the second column of the table. These are the charges carried by the strings in the $d$-dimensional theory and we will denote them by the column vector $Z^{a}$. Keeping the $E_{11}$ fields still only at level zero the generalised vielbein takes the from

$$
E=(\operatorname{det} e)^{-\frac{1}{2}}\left(\begin{array}{ccc}
e_{\mu}{ }^{a} & 0 & 0 \\
0 & \mathcal{E}^{(0)} & 0 \\
0 & 0 & \mathcal{E}^{(1)}
\end{array}\right)
$$

and so the half BPS condition of equation (12) takes the form

$$
p^{2}+Z^{T}\left(\mathcal{E}^{(0) T}\right)^{-1}\left(\mathcal{E}^{(0)}\right)^{-1} Z+Z^{a T}\left(\mathcal{E}^{(1) T}\right)^{-1}\left(\mathcal{E}^{(1)}\right)^{-1} Z_{a}=0
$$

It is instructive to explicitly evaluate this condition for the seven dimensional theory. The charges at level one belong to the $\overline{5}$ representations of SL(5) and as seen from the view point of eleven dimensions they arise as $Z^{a}=\left\{Z^{a i}, Z^{a i_{1} \ldots i_{4}}\right\}$ where $i, i_{1}, \ldots=1,2,3,4$. To compute the generalised vielbein at level one we consider

$$
(d x \cdot l)^{(1)}=d x_{a i} Z^{a i}+d x_{a i_{1} i_{2}} Z^{a i_{1} i_{2}}
$$

Using equation (9) and the commutators of the appendix, we find that the generalised vielbein at level one in the internal sector is given by

$$
\mathcal{E}^{(1)}=\left(\begin{array}{cc}
\left(e^{-1}\right)_{j}^{\bar{i}} & -\frac{1}{3 !}\left(e^{-1}\right)_{\left[j_{1}\right.}{ }^{\overline{ }} A_{\left.j_{2} j_{2} j_{3}\right]} \\
0 & \left(e^{-1}\right)_{j_{1} j_{2} j_{3} j_{4}} \bar{i}_{1} \bar{i}_{2} \bar{i}_{3} \bar{i}_{4}{ }
\end{array}\right),
$$

with the inverse

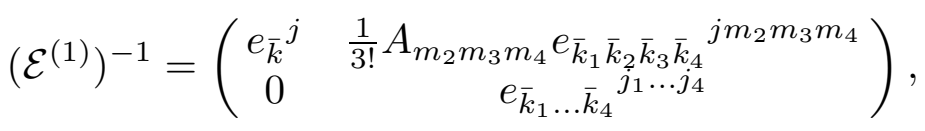

Substituting into equation (28) one finds the BPS condition. For situations in which the point particle charges vanish one finds a constraint for just the string charges.

The generalisations to include higher levels in the $l_{1}$ representation, and indeed higher levels in the $E_{11}$ fields, is straightforward and the half BPS condition can be readily computed, at least at low levels.

We will now evaluate the $E_{11}$ half BPS condition of equations (12) and (13) in the familiar context of ten dimensions at level zero. The non-linear realisation of $E_{11} \otimes_{s} l_{1}$, first advocated in [10], was carried out in in this context in reference [27]. It is just contains the fields of the IIA NS-NS sector but they depend on the coordinates $x^{a}$ and $y_{a}$ which transform as the vector representation of $\mathrm{O}(10,10)$. It is nothing but the so called doubled 
field theory [28]. The level one non-linear realisation contains the fields in the R-R sector [29]. To find the IIA theory in ten dimensions one deletes node ten in the $E_{11}$ Dynkin diagram to find the Dynkin diagram of the algebra $\mathrm{SO}(10,10)$ and so one must decompose $E_{11}$ into representations of $\mathrm{SO}(10,10)$. The non-linear realisation of $E_{11}$ at lowest level contains the fields of the NS-NS sector, namely $h_{a}{ }^{b}, A_{a b}$ and $a$, while the $l_{1}$ representation contains the charges $P_{a}$ and $P^{\bar{a}}$ which form the vector representation of $\mathrm{O}(10,10)$. The corresponding non-linear realisation, and so the dynamics, was worked out in detail at level zero in [27] and level one in [29]. In the first paper we denoted the level zero generators in the $l_{1}$ representation by the symbols $P_{a}$ and $Q^{a}$ while in the second paper, and in this paper, we use the same $P_{a}$ but $P^{\bar{a}}=\frac{1}{2} Q^{a}$. In terms of the eleven-dimensional charges $Z^{a 11}=-P^{\bar{a}}$.

The generalised vielbein, at lowest level, was found [27, 29] to be given by

$$
E_{N}{ }^{A}=(\operatorname{det} e)^{-\frac{1}{2}}\left(\begin{array}{cc}
e & e A e^{-\frac{1}{2} a} \\
0 & e^{-1 T} e^{-\frac{1}{2} a}
\end{array}\right)
$$

and so the charges referred to the tangent space take the form

$$
L_{A}=\left(E^{-1}\right)_{A}{ }^{N} l_{N}=(\operatorname{det} e)^{\frac{1}{2}}\left(\begin{array}{c}
\left(e^{-1}\right){ }_{a}^{\mu}{ }^{\mu} P_{\mu}-e^{\frac{a}{2}} A_{a \nu} P^{\bar{\nu}} \\
e^{\frac{a}{2}} e_{\mu}{ }^{a} P^{\bar{\mu}}
\end{array}\right)
$$

As such the half BPS condition of equation (12) becomes

$$
L^{2}=L_{A} L_{A}=\left(P_{\mu}, P^{\bar{\mu}}\right) M^{-1}\left(\begin{array}{c}
P_{\mu} \\
P^{\bar{\mu}}
\end{array}\right)=0
$$

where $M=E E^{T}$. The other half BPS constraint of equation (13) takes the simple form

$$
P_{\mu} P^{\bar{\mu}}=0
$$

If we consider the ten dimensional string to be compactified on a torus then the Kaluza-Klein momenta and winding modes are identified with $P_{i}$ and $P^{\bar{i}}$ respectively. As a result, suppressing the radii of the tori, we must set these equal to the integers $m$ and $n$ respectively. In this case the half BPS condition of equation (34) becomes by

$$
p^{2}+(m, n) M^{-1}\left(\begin{array}{c}
m \\
n
\end{array}\right)=0
$$

where $p_{\mu}$ is the momentum in the uncompactified space-time and the second condition of equation (35) takes the form

$$
m \cdot n=0
$$

We recognise equations (36) and (37) as the well known half BPS conditions for the compactified string.

We now consider the conditions if only a quarter of the original supersymmetry survives from and $E_{11}$ viewpoint. Examining the supersymmetry algebra one finds that these 
conditions are quartic in the central charges, see [19] for a very telegraphed account. Let us define

$$
\left.s_{10} \equiv l \otimes l\right|_{l_{10}}
$$

The quarter BPS condition should revert to the half BPS conditions if $s_{10}=0$ and so we propose that quarter BPS states should satisfy

$$
\left(l^{T} D\left(M^{-1}\right) l\right)^{2}=s_{10}^{T} D\left(M^{-1}\right) s_{10}
$$

Now the matrix $D$ is that appropriate to the representation on which it acts. Guided by an analysis of the supersymmetry algebra one finds that if $s_{10}$ does not vanishes then demanding a quarter supersymmetry leads to the constraints

$$
S^{\left[a_{1}\right.} S^{\left.a_{2} \ldots a_{5}\right]}=0, S^{\left[a_{1} \ldots a_{4}\right.} S^{\left.a_{1} \ldots a_{4}\right]}=0, \ldots
$$

As such one should also insist on the condition

$$
\left.s_{10} \otimes s_{10}\right|_{l_{6}}=0
$$

that is, the tensor product of the two $s_{10}$ representations, each of which is constructed from the two $l_{1}$ representations, when projected in the first fundamental representation associated with node six, denoted $l_{6}$, vanishes. It is straightforward to evaluate these conditions at lowest level.

In this paper we have proposed two $E_{11}$ conditions that when evaluated at lowest level agree with the known half BPS conditions. We have not derived these conditions from first principles, that is supersymmetry preservation. However, they are the unique $E_{11}$ invariant conditions which are bilinear in the brane charges and in the case of the second condition of equation (13) also have a first component that is an object with one upper SL(11) index. The conditions are very simple and easy to evaluate. These $E_{11}$ conditions contain an infinite number of fields and charges in addition to those with which people are familiar, however, there is substantial evidence that many of these objects are required in an underlying theory of strings and branes and as such the conditions found in this paper provide the required generalisations of the known BPS conditions.

This paper provides another example where $E_{11}$ predicts results that are traditionally thought to be the result of supersymmetry. It would be good to understand at a deeper level what is the connection between $E_{11}$ and supersymmetry.

It was not clear from the outset that the brane charges in all dimensions should assemble into one $E_{11}$ multiplet, but we now know that this is the case and the multiplet in question is the $l_{1}$ multiplet. In this paper we have seen that half BPS conditions for all the charges, that is, space-time momentum, particle, string, etc, assemble into simple $E_{11}$ equations. This can be interpreted as evidence of an underlying $E_{11}$ symmetry.

The one part of doubled field theory [28] that is not contained in the non-linear realisation of $E_{11} \otimes_{s} l_{1}$ at lowest level, as first proposed in [10], is the so called section condition, which is essentially equation (32). It has been found that in most circumstances this just sets to zero the dependence on one coordinates. A generalisation of this condition 
to the type of generalised space-time at lowest level first proposed in [10], was given in reference [30]. This is essentially the condition of equation (13), but applied to the usual U duality groups $E_{n}, n=4,5,6,7,8$. We see that this condition is really one of the half BPS conditions, but one might expect that the theory will involve more than just half BPS states. One could for example impose the more general quarter BPS conditions of equation (41). A first principle, although limited, approach to the question of how to reduce the generalised space-time to something we recognise was given in [31].

We end with a speculative comment on higher derivative string corrections and the automorphic forms that describe them. It has been observed that the automorphic forms that appear in front of the $R^{4}$ and $D^{4} R$ terms in type II string theory can be derived from the eleven dimensional one [32] and two [33] loop diagrams respectively. The sums over the integers arising from the Kaluza-Klein and other half BPS modes. In particular the auotomorphic form for the $R^{4}$ term arises from a scalar-like one loop box diagram at zero external momentum which in nine dimensions is of the generic form

$$
\sum_{n_{1}, n_{2}} \int d^{9} k \frac{1}{\left(k^{2}+M\left(n_{1}, n_{2}\right)^{2}\right)^{4}}
$$

where $M\left(n_{1}, n_{2}\right)^{2}$ is the mass of the Klauza-Klein modes as it appears in the half BPS condition. For lower dimensions one should also impose the half BPS conditions of equation (21). There is now overwhelming evidence that this automorphic form correctly accounts for all the $R^{4}$ corrections.

We note that $L^{-2}$ looks like a propagator with a mass that is one of the BPS states; it is also $E_{11}$ invariant. If we were to take into account all the BPS states that should occur in the underlying theory, then we would expect to find a coefficient for the $R^{4}$ term of the generic form

$$
\sum \frac{1}{\left(l^{2}\right)^{4}}
$$

The sum is to be interpreted as being over the half BPS charges that are active and this will depend on the dimension one is in. The sum will include an integral over the space-time momentum in the uncompactified space-time and where appropriate the Kaluza Klein modes (internal momentum components), the winding modes (components of the rank two central charge), etc. If we include only low levels then the object in equation (43) will lead to the automorphic forms that arise in equation (42). However, at higher levels one finds terms arising from the fact that one has an $E_{11}$ automorphic form. A method of constructing automorphic forms for any group was given in $[22,35]$ and although this was applied to find the correct automorphic forms for the usual finite $U$ duality groups it can also be applied to $E_{11}$, so leading also to the form given generically in equation (43). We note that one must also by hand implement the half BPS conditions of equation (13) on the automorphic form.

The $R^{4}$ part of the correction together with other terms constructed from bosonic fields can hopefully be constructed from $E_{11}$ Cartan forms in such a way so as to be $E_{11}$ invariant. This is to be expected as terms related by supersymmetry should have the same automorphic form. Thus the effective action should be composed of different $E_{11}$ invariant 
building blocks. From the $E_{11}$ perspective the different theories arise by taking different decompositions of $E_{11}$ and as a result they all have a common origin. An $E_{11}$ formulation of the higher string corrections would encode this common origin. This is consistent with the fact that the BPS masses that appear in equation (42) are the same regardless of whether they are computed from the IIB, IIA or M theory perspectives [36]. Further ideas concerning how higher derivative string corrections can admit an $E_{11}$ formulation can be found in [37].

\section{Acknowledgment}

This work reported in this paper began with a number of discussions between the author, David Berman and Malcolm Perry at Cooks Branch Conservancy, Texas; I thank them for their help at an early stage of this work. Notes containing the results in this paper were circulated to a small set of people in Cambridge, England in the summer of 2011. I wish to thank the STFC for support from grant number ST/J002798/1 and the George Mitchell Foundation for funding for the visit to Cooks Branch Conservancy.

\section{Appendix The $E_{11} \otimes_{s} l_{1}$ algebra}

This appendix is designed to equip the reader with the $E_{11}$ material required to understand this paper. Rather than explain the theory behind Kac-Moody algebras we will present the required results. We first give the $E_{11}$ algebra in the decomposition appropriate to eleven dimensions, that is, we decompose the $E_{11}$ algebra into representations of $A_{10}$, or SL(11), representations [4,10]. This algebra is found by deleting node eleven in the Dynkin Diagram. The way one constructs the $E_{11}$ algebra, from the definition of $E_{11}$ as a Kac-Moody algebra, in terms of representations of SL(11) is discussed, for example, in [21]. For the calculation in this paper one does not need to understand all the subtleties of this construction and the parts of the algebra that are needed are given below. The generators can be classified according to a level which is associated with the decomposition associated with the deletion of node eleven. At level zero we have the algebra GL(11) with the generators $K_{b}^{a}, a, b=1, \ldots 11$ and at level one and minus one the rank three generators $R^{a b c}$ and $R_{a b c}$ respectively. The generators at level two and minus two are $R^{a_{1} \ldots a_{6}}$ and $R_{a_{1} \ldots a_{6}}$ respectively, while those at levels three and minus three are $R^{a_{1} \ldots a_{8}, b}$ and $R_{a_{1} \ldots a_{8}, b}$ respectively. The level is just the number of upper minus lower indices divided by three. For a discussion giving the more abstract definition of level which relates it to the deletion of node eleven see for example reference [21].

The $E_{11}$ algebra at levels zero and up three is given by $[4,10]$

$$
\begin{gathered}
{\left[K_{b}^{a}, K_{d}^{c}\right]=\delta_{b}^{c} K_{d}^{a}-\delta_{d}^{a} K_{b}^{c},} \\
{\left[K_{b}^{a}, R^{c_{1} \ldots c_{6}}\right]=\delta_{b}^{c_{1}} R^{a c_{2} \ldots c_{6}}+\ldots,\left[K_{b}^{a}, R^{c_{1} \ldots c_{3}}\right]=\delta_{b}^{c_{1}} R^{a c_{2} c_{3}}+\ldots,} \\
{\left[K_{b}^{a}, R^{c_{1} \ldots c_{8}, d}\right]=\left(\delta_{b}^{c_{1}} R^{a c_{2} \ldots c_{8}, d}+\ldots\right)+\delta_{b}^{d} R^{c_{1} \ldots c_{8}, a} .}
\end{gathered}
$$

and

$$
\left[R^{c_{1} \ldots c_{3}}, R^{c_{4} \ldots c_{6}}\right]=2 R^{c_{1} \ldots c_{6}}, \quad\left[R^{a_{1} \ldots a_{6}}, R^{b_{1} \ldots b_{3}}\right]=3 R^{a_{1} \ldots a_{6}\left[b_{1} b_{2}, b_{3}\right]},
$$

where $+\ldots$ means the appropriate anti-symmetrisation. 
The $E_{11}$ level zero and negative level generators up to level minus three obey the relations

$$
\begin{gathered}
{\left[K_{b}^{a}, R_{c_{1} \ldots c_{3}}\right]=-\delta_{c_{1}}^{a} R_{b c_{2} c_{3}}-\ldots,\left[K_{b}^{a}, R_{c_{1} \ldots c_{6}}\right]=-\delta_{c_{1}}^{a} R_{b c_{2} \ldots c_{6}}-\ldots,} \\
{\left[K_{b}^{a}, R_{c_{1} \ldots c_{8}, d}\right]=-\left(\delta_{c_{1}}^{a} R_{b c_{2} \ldots c_{8}, d}+\cdots\right)-\delta_{d}^{a} R_{c_{1} \ldots c_{8}, b}} \\
{\left[R_{c_{1} \ldots c_{3}}, R_{c_{4} \ldots c_{6}}\right]=2 R_{c_{1} \ldots c_{6}}, \quad\left[R_{a_{1} \ldots a_{6}}, R_{b_{1} \ldots b_{3}}\right]=3 R_{a_{1} \ldots a_{6}\left[b_{1} b_{2}, b_{3}\right]}}
\end{gathered}
$$

Finally, the commutation relations between the positive and negative generators are given by

$$
\begin{aligned}
& {\left[R^{a_{1} \ldots a_{3}}, R_{b_{1} \ldots b_{3}}\right]=18 \delta_{\left[b_{1} b_{2}\right.}^{\left[a_{1} a_{2}\right.} K^{\left.a_{3}\right]}{ }_{\left.b_{3}\right]}-2 \delta_{b_{1} b_{2} b_{3}}^{a_{1} a_{2} a_{3}} D,\left[R_{b_{1} \ldots b_{3}}, R^{a_{1} \ldots a_{6}}\right]=\frac{5 !}{2} \delta_{b_{1} b_{2} b_{3}}^{\left[a_{1} a_{2} a_{3}\right.} R^{\left.a_{4} a_{5} a_{6}\right]}} \\
& {\left[R^{a_{1} \ldots a_{6}}, R_{b_{1} \ldots b_{6}}\right]=-5 ! .3 .3 \delta_{\left[b_{1} \ldots b_{5}\right.}^{\left[a_{1} \ldots a_{5}\right.} K^{\left.a_{6}\right]}{ }_{\left.b_{6}\right]}+5 ! \delta_{b_{1} \ldots b_{6}}^{a_{1} \ldots a_{6}} D} \\
& {\left[R_{a_{1} \ldots a_{3}}, R^{b_{1} \ldots b_{8}, c}\right]=8.7 .2\left(\delta_{\left[a_{1} a_{2} a_{3}\right.}^{\left[b_{1} b_{2} b_{3}\right.} R^{\left.b_{4} \ldots b_{8}\right] c}-\delta_{\left[a_{1} a_{2} a_{3}\right.}^{\left[b_{1} b_{2}|c|\right.} R^{\left.b_{3} \ldots b_{8}\right]}\right)} \\
& {\left[R_{a_{1} \ldots a_{6}}, R^{b_{1} \ldots b_{8}, c}\right]=\frac{7 ! .2}{3}\left(\delta_{\left[a_{1} \ldots a_{6}\right.}^{\left[b_{1} \ldots b_{6}\right.} R^{\left.b_{7} b_{8}\right] c}-\delta_{\left[a_{1} \ldots a_{6}\right.}^{c\left[b_{1} \ldots b_{5}\right.} R^{\left.b_{6} b_{7} b_{8}\right]}\right)}
\end{aligned}
$$

where $D=\sum_{b} K_{b}^{b}, \delta_{b_{1} b_{2}}^{a_{1} a_{2}}=\frac{1}{2}\left(\delta_{b_{1}}^{a_{1}} \delta_{b_{2}}^{a_{2}}-\delta_{b_{1}}^{a_{2}} \delta_{b_{2}}^{a_{1}}\right)=\delta_{b_{1}}^{\left[a_{1}\right.} \delta_{b_{2}}^{\left.a_{2}\right]}$ with similar formulae when more indices are involved.

We also need the fundamental representation of $E_{11}$ associated with node one, denoted by $l_{1}$. By definition this is the representation with highest weight $\Lambda_{1}$ which obeys $\left(\Lambda_{1}, \alpha_{a}\right)=\delta_{a, 1}, a=1,2 \ldots, 11$ where $\alpha_{a}$ are the simple roots of $E_{11}$. In the decomposition to $\mathrm{Sl}(11)$, corresponding to the deletion of node eleven, one finds that the $l_{1}$ representation contains the objects $P_{a}, Z^{a b}$ and $Z^{a_{1} \ldots a_{5}}, a, b, a_{1}, \ldots=1, \ldots, 11$ corresponding to levels zero, one and two respectively. We have taken the first object, i.e. $P_{a}$, to have level zero by choice. Taking these to be generators belong to a semi-direct product algebra with those of $E_{11}$, denoted by $E_{11} \otimes_{s} l_{1}$, their commutation relations with the level one generators of $E_{11}$ are given by [10]

$$
\begin{gathered}
{\left[R^{a_{1} a_{2} a_{3}}, P_{b}\right]=3 \delta_{b}^{\left[a_{1}\right.} Z^{\left.a_{2} a_{3}\right]},\left[R^{a_{1} a_{2} a_{3}}, Z^{b_{1} b_{2}}\right]=Z^{a_{1} a_{2} a_{3} b_{1} b_{2}},} \\
{\left[R^{a_{1} a_{2} a_{3}}, Z^{b_{1} \ldots b_{5}}\right]=Z^{b_{1} \ldots b_{5}\left[a_{1} a_{2}, a_{3}\right]}+Z^{b_{1} \ldots b_{5} a_{1} a_{2} a_{3}}}
\end{gathered}
$$

These equations define the normalisation of the generators of the $l_{1}$ representation. The commutators of the generators of the $l_{1}$ representation with those of GL(11) are given by

$$
\begin{gathered}
{\left[K_{b}^{a}, P_{c}\right]=-\delta_{c}^{a} P_{b}+\frac{1}{2} \delta_{b}^{a} P_{c},\left[K_{b}^{a}, Z^{c_{1} c_{2}}\right]=2 \delta_{b}^{\left[c_{1}\right.} Z^{\left.|a| c_{2}\right]}+\frac{1}{2} \delta_{b}^{a} Z^{c_{1} c_{2}}} \\
{\left[K^{a}{ }_{b}, Z^{c_{1} \ldots c_{5}}\right]=5 \delta_{b}^{\left[c_{1}\right.} Z^{\left.|a| c_{2} \ldots c_{5}\right]}+\frac{1}{2} \delta_{b}^{a} Z^{c_{1} \ldots c_{5}}}
\end{gathered}
$$


The commutation relations with the level two generators of $E_{11}$ are given by

$$
\left[R^{a_{1} \ldots a_{6}}, P_{b}\right]=-3 \delta_{b}^{\left[a_{1}\right.} Z^{\left.\ldots a_{6}\right]},\left[R^{a_{1} \ldots a_{6}}, Z^{b_{1} b_{2}}\right]=Z^{b_{1} b_{2}\left[a_{1} \ldots a_{5}, a_{6}\right]}
$$

The commutators with the level -1 negative root generatorsare given by

$$
\left[R_{a_{1} a_{2} a_{3}}, P_{b}\right]=0,\left[R_{a_{1} a_{2} a_{3}}, Z^{b_{1} b_{2}}\right]=6 \delta_{\left[a_{1} a_{2}\right.}^{b_{1} b_{2}} P_{\left.a_{3}\right]},\left[R_{a_{1} a_{2} a_{3}}, Z^{b_{1} \ldots b_{5}}\right]=\frac{5 !}{2} \delta_{a_{1} a_{2} a_{3}}^{\left[b_{1} b_{2} b_{3}\right.} Z^{\left.b_{4} b_{5}\right]}
$$

\section{References}

[1] E. Witten and D. Olive, Supersymmetry algebras that include topoloogical charges, Phys. Lett. 78B (1978) 97.

[2] J. Azcarraga, J. Gauntlett, J. Izquierdo and P. Townsend, Topological Extensions of the Supersymmetry Algebra for Extended Objects, Phys. Rev. Lett. 63, no 22 (1989) 2443.

[3] S. Ferrra and J Maldecena, Branes, central charges and U-duality invariant BPS conditions, arXiv:hep-th/9706097; B. Pioline and E Kiritsis, U-duality and D-brane Combinatorics, Phys.Lett. B418 (1998) 61, arXiv:hep-th/9710078.

[4] P. West, $E_{11}$ and $M$ Theory, Class. Quant. Grav. 18 (2001) 4443, arXiv:hep-th/ 0104081

[5] I. Schnakenburg and P. West, Kac-Moody symmetries of IIB supergravity, Phys. Lett. B517 (2001) 421, arXiv:hep-th/0107181.

[6] F. Riccioni and P. West, The $E_{11}$ origin of all maximal supergravities, JHEP 0707 (2007) 063; arXiv:0705.0752.

[7] F. Riccioni and P. West, E(11)-extended spacetime and gauged supergravities, JHEP 0802 (2008) 039, arXiv:0712.1795

[8] F. Riccioni and P. West, Local E(11), JHEP 0904 (2009) 051, arXiv:hep-th/0902.4678.

[9] F. Riccioni, D. Steele and P. West, The E(11) origin of all maximal supergravities the hierarchy of field-strengths JHEP 0909 (2009) 095, arXiv:0906.1177.

[10] P. West, $E_{11}$, SL(32) and Central Charges, Phys. Lett. B 575 (2003) 333-342, hepth/0307098

[11] A. Kleinschmidt and P. West, Representations of $G+++$ and the role of space-time, JHEP 0402 (2004) 033, hep-th/0312247.

[12] P. West, E $E_{11}$ origin of Brane charges and U-duality multiplets, JHEP 0408 (2004) 052, hep-th/0406150.

[13] P. Cook and P. West, Charge multiplets and masses for E(11), JHEP 11 (2008) 091, arXiv:0805.4451.

[14] P. West, The IIA, IIB and eleven dimensional theories and their common $E_{11}$ origin, Nucl. Phys. B693 (2004) 76-102, hep-th/0402140.

[15] S. Elitzur, A. Giveon, D. Kutasov and E. Rabinovici, Algebraic aspects of matrix theory on $T^{d}$, arXiv:hep-th/9707217.

[16] N. Obers, B. Pioline and E. Rabinovici, M-theory and U-duality on $T^{d}$ with gauge backgrounds, hep-th/9712084

[17] N. Obers and B. Pioline, U-duality and M-theory, an algebraic approach, hepth/9812139. 
[18] B. de Wit and H. Nicolai, Hidden symmetries, central charges and all that, hepth/0011239.

[19] N. Obers and B. Pioline, U-duality and M-theory, arXiv:hep-th/9809039.

[20] P. West, Brane dynamics, central charges and $E_{11}$, hep-th/0412336.

[21] P. West,Introduction to Strings and Branes, Cambridge University Press, June 2012.

[22] P. West and N. Lambert, Duality Groups, Automorphic Forms and Higher Derivative Corrections, Phys.Rev. D75 (2007) 066002, hep-th/0611318

[23] T. Damour, M. Henneaux and H. Nicolai, E(10) and a 'small tension expansion' of $M$ theory, Phys. Rev. Lett. 89 (2002) 221601, arXiv:hep-th/0207267.

[24] P. West, Very Extended $E_{8}$ and $A_{8}$ at low levels, Gravity and Supergravity, Class.Quant.Grav. 20 (2003) 2393, hep-th/0212291.

[25] T. Nutma, SimpLie, a simple program for Lie algebras, https://code.google.com/p/simplie/.

[26] D. Berman, H. Godazgar, M. Perry and P. West, Duality Invariant Actions and Generalised Geometry, arXiv:1111.0459.

[27] P. West, E11, generalised space-time and IIA string theory, Phys.Lett.B696 (2011) 403-409, arXiv:1009.2624.

[28] O. Hohm, C. Hull and B. Zwiebach, Generalised metric formulation of double field theory, hep-th/1006.4823;

[29] A. Rocen and P. West, E11, generalised space-time and IIA string theory; the $R$ - $R$ sector, arXiv:1012.2744.

[30] A. Coimbra, C. Strickland-Constable and D. Waldram, $E_{d(d)} \times R^{+}$Generalised Geometry, Connections and $M$ theory, arXiv:1112.3989.

[31] P. West, Generalised space-time and duality, hep-th/1006.0893.

[32] M.B. Green, M. Gutperle and P. Vanhove, One loop in eleven dimensions, Phys.Lett. B409 (1997) 177, arXiv:hep-th/9706175.

[33] J. Russo and A.A. Tseytlin, One-loop four-graviton amplitude in eleven-dimensional supergravity, Nucl.Phys. B508 (1997) 245, arXiv:hep-th/9707134.

[34] Michael B. Green and Hwang-h. Kwon, Pierre Vanhove, Two loops in eleven dimensions, Phys.Rev. D61(2000) 104010, arXiv:hep-th/9910055.

[35] N. Lambert and P. West, Perturbation Theory From Automorphic Forms, JHEP 1005 (2010) 098, arXiv:1001.3284.

[36] J. Schwarz, An SL(2,Z) Multiplet of Type IIB Superstrings, Phys.Lett. B360 (1995) 13; Erratum-ibid. B364 (1995) 252, arXiv:hep-th/9508143; B, de Wit and D. Lust, BPS Amplitudes, Helicity Supertraces and Membranes in M-Theory Journal-ref: Phys.Lett.B477 (2000) 299, arXiv:hep-th/9912225

[37] F. Gubay and P. West, Higher derivative type II string effective actions, automorphic forms and E11, arXiv:1111.0464. 